\title{
Inflammatory Mediators in Diabetic Neuropathy
}

Natalie M. Wilson and Douglas E. Wright*

Department of Anatomy and Cell Biology, University of Kansas Medical Center, Kansas City, KS 66160

\begin{abstract}
Diabetic neuropathy (DN) is the most common complication of diabetes mellitus, resulting in high health care costs and greatly affecting the quality of life of patients. The mechanism(s) responsible for the development of DN is currently unclear, but is likely multifactoral. While a great deal is still unknown about the mediators responsible for the development of DN, current knowledge of other neuropathic conditions suggest the involvement of a number of inflammatory mediators such as interleukins and chemokines. This review will outline the current literature of a number of inflammatory mediators in both human and rodent studies. As there is currently no therapeutic treatment for DN, this review will also provide an overview of therapeutics targeting inflammation and the ability of these therapies to improve the symptoms of DN.
\end{abstract}

\section{Introduction}

Diabetes mellitus currently affects 25.8 million individuals in the United States with total costs in the US reaching $\$ 174$ billion (www. diabetes.org). Diabetes is associated with a number of complications including: heart disease, stroke, blindness, kidney disease, and neuropathy. Diabetic neuropathy (DN) is the most common complication of diabetes, with $60-70 \%$ of diabetic patients reporting mild to severe forms of peripheral nerve dysfunction (www.diabetes. org). DN is characterized by a number of neural symptoms including numbness, sensory loss, and stabbing or burning pain typically experienced in the hands and feet. These sensory symptoms are thought to associate with the progressive loss or damage to sensory nerve fibers. A number of mechanisms for $\mathrm{DN}$ have been proposed, ranging from increased reactive oxygen species production, increased protein glycosylation, neurovascular disturbances, and decreased neurotrophic support [1]. One area that has had comparatively limited investigation is the role of inflammatory mediators in DN. Inflammatory mediators such as cytokines have been linked to many forms of neuropathic pain. Thus, the role that cytokines play in DN should merit exploration and will be the focus of this review.

\section{Nuclear Factor Kappa B (NF-kB)}

$\mathrm{NF}-\kappa \mathrm{B}$ is a transcriptional factor that is activated by a number of stimuli and is responsible for initiating the transcription of a number of different inflammatory and immune mediators. Therefore, monitoring the activation of this transcription factor can be used as an indicator of the state of the inflammation in tissue. Analyzing various tissues from diabetic patients has revealed increased NF- $\kappa \mathrm{B}$ activity in the kidney, endothelial cells, peripheral blood mononuclear cells, monocytes, and sural nerve [2-5]. Further characterization has been conducted in rodent studies from which NF- $\kappa \mathrm{B}$ has been identified as a factor involved in models of diabetic neuropathy. NF- $\kappa B$ activation is increased in the dorsal root ganglion (DRG), sural, and sciatic nerve of diabetic mice compared to non-diabetic mice $[3,6]$. Investigations into the mechanism of increased NF- $\mathrm{kB}$ activation have demonstrated that NF- $\mathrm{KB}$ activation could be prevented in receptor for advanced glycation end products (RAGE) null mutant mice [6]. The involvement of glycosylated proteins and RAGE has been explored in a number of complications of diabetes, but whether RAGE signaling also leads to inflammatory changes needs further exploration.

\section{Toll-Like Receptors}

Toll like receptors (TLR) are pattern recognition receptors utilized by the innate immune system. Activation of TLRs induces NF- $\kappa B$ activation and increases the expression of a number of cytokines and chemokines. Two of the most highly characterized toll-like receptors, TLR2 and TLR4, are expressed in a number of cell types including monocytes. High glucose treatment of human monocytic cell lines increases the expression of TLR2 and TLR4 in a dose-dependent manner. Additionally, high glucose treatment of these cells also leads to increased release of IL-1, IL-6, MCP-1, and TNF- $\alpha$, which is prevented in TLR2 and TLR4 knockdown conditions [7]. Follow-up studies utilizing monocytes from human patients further confirmed the in vitro findings. Monocyte TLR2 and TLR4 surface expression and mRNA levels were significantly increased from Type 1 Diabetic patients compared to healthy controls [5]. This group went on to demonstrate significantly higher circulating levels of TLR2 and TLR4 ligands, highmobility group box 1 (HMGB1) and heat shock protein 60 (Hsp60), in Type 1 patients compared to control patients [8].

While changes in TLRs have been demonstrated in diabetic patients, the link between those changes and DN is just beginning to be explored. Two common single nucleotide polymorphisms exist in the TLR4 gene at Asp299Gly and Thr399Ile. The presence of these polymorphisms alters the structure of the extracellular domain, which has the potential to alter ligand binding [9]. Analysis of the TLR4 gene in diabetic patients revealed that the presence of Asp299Gly and Thr399Ile genotypes reduced the incidence of DN in Type 2 patients. While the TLR4 polymorphisms also occurred in Type 1 patients the protection against the development of DN was not witnessed [10]. However, the number of Type 1 patients in the study was lower than Type 2 patients which might alter the outcome. The possible mechanism behind the protection of these polymorphisms against the development of DN could stem from changing the extracellular structure of TLR4 and potentially altering the ligand binding domain.

*Corresponding author: Douglas Wright, PhD, Department of Anatomy and Cel Biology, University of Kansas Medical Center, Kansas City, KS 66160, Tel: 913 588-2713; Fax: 913-588-2710; E-mail: dwright@kumc.edu

Received November 11, 2011; Accepted December 17, 2011; Published December 22, 2011

Citation: Wilson NM, Wright DE (2011) Inflammatory Mediators in Diabetic Neuropathy. J Diabetes Metab S5:004. doi:10.4172/2155-6156.S5-004

Copyright: (C) 2011 Wilson NM, et al. This is an open-access article distributed under the terms of the Creative Commons Attribution License, which permits unrestricted use, distribution, and reproduction in any medium, provided the original author and source are credited. 
Combining this data with the presence of increased TLR4 ligands could explain this protection, by preventing the increased inflammatory response induced by TLR4 signaling.

\section{Cytokines}

\section{Interleukins}

Interleukins (IL) are a group of cytokines named for their ability to communicate between leukocytes. In recent years, research has shown their involvement in cell signaling in a number of other cell types and tissues. There are more than 30 interleukin isoforms currently identified that can have either pro-inflammatory (IL-1,IL-6 and IL-8) or anti-inflammatory (IL-4 and IL-10) actions. Interleukins have been shown to be involved in a number of different neuropathic conditions in both animal and human studies. Clinical studies in small-fiber neuropathy patients demonstrated a two-fold increase in circulating IL-2 mRNA levels in peripheral blood compared to healthy controls. Skin samples from the affected area were collected from patients, which showed increased IL- 6 and IL-8 mRNA levels compared to controls. Further analysis comparing the affected skin area to unaffected skin areas in this patient population revealed increases in IL-1 $\beta$, IL-6, and IL-8 mRNA levels, 2-fold, greater than 200 fold, and greater than 500 fold, respectively [11]. While significant research has been done in other neuropathic conditions, research on the role interleukins play in diabetic neuropathy is just beginning to be explored. Rodent studies utilizing streptozotocin (STZ) injections to induce DN have revealed increased in IL-6 mRNA levels in both the DRG and sciatic nerve compared to non-diabetic mice $[3,12]$. Clinical findings utilizing sural nerve biopsies from DN patients demonstrated increased IL-6 expression compared to healthy controls [3]. Expansion of these findings with particular focus on how interleukins contribute to the symptoms of DN such as nerve conduction velocity and fiber loss is still needed.

\section{Chemokines}

Chemotactic cytokines, or chemokines, are an integral part of the immune system and in recent years have been shown to be involved in nociceptive behavior. The role of chemokines in nociceptive behavior was been demonstrated through hindpaw injections of chemokines, stromal derived factor 1 (SDF1) and regulated on activation normally T-cell expressed and secreted (RANTES), inducing decreases in mechanical threshold [13]. The chemokines role in neuropathic pain states has also been shown in both drug- and injury- induced neuropathy rodent models, such as anti-HIV treatment and chronic constriction injury [14]. Chemokines role in diabetic neuropathy has begun to be explored as well. Rodent studies have demonstrated monocyte chemoattractant protein-1 (MCP-1) is increased in both plasma and adipose tissue in diabetic ob/ob mice compared to nondiabetic lean mice [15]. Clinical studies utilizing serum samples from diabetic patients have demonstrated significant increases in MCP-1 and RANTES levels compared to controls [16,17]. Guan and colleagues expanded on their findings by revealing a significant correlation between increases in serum MCP-1 levels and diabetic complications, including peripheral neuropathy [17]. The link between DN and chemokines was strengthened by reports of significantly increased levels of circulating RANTES in DN patients when compared to healthy controls and diabetic patients with no symptoms of neuropathy [18]. While the association between chemokines and DN is correlative at this time, given the demonstrated role chemokines play in other neuropathies, further exploration is warranted, including changes in chemokine expression within the peripheral nervous system.

\section{Tumor Necrosis Factor $\alpha($ TNF- $\alpha)$}

TNF- $\alpha$ is a pro-inflammatory cytokine that has been implicated in neuropathic and inflammatory nociceptive conditions for a number of years. This link stems from the ability of TNF- $\alpha$ administration to induce ectopic firing in sensory neurons [19] and mechanical and thermal nociceptive behavior [20] in rodents. More confirmation of the link between TNF- $\alpha$ and nociceptive behavior is evidenced by the fact that TNF- $\alpha$ mRNA expression corresponds to the time course of the development of thermal and mechanical behavior in a neuropathic pain model using chronic constriction injury [21].

Recently TNF- $\alpha$ has also begun to be implicated in diabetic neuropathy. Clinical studies have shown increased TNF- $\alpha$ plasma protein and mRNA levels in diabetic patients compared to controls [22-24]. Increased TNF- $\alpha$ macrophage expression and plasma levels were also demonstrated in $\mathrm{DN}$ patients compared to controls and was correlated to pain intensity [22]. Exploration of these clinical findings in rodent models has been key to understanding its role in DN. STZinduced DN in rodents results in increased circulating levels of TNF- $\alpha$ $[25,26,27,28]$ and the consequence of increased TNF- $\alpha$ expression was explored in STZ-injected rats. TNF- $\alpha$ administration into the sciatic nerve induced a reduction in motor nerve conduction velocity (MNCV), a symptom commonly witnessed in patients with DN [29]. TNF-a's role in the symptoms of DN has been validated in studies utilizing TNF- $\alpha$ null mutant mice. TNF- $\alpha$ deficient diabetic mice fail to develop changes in nociceptive behavior, MNCV, and sensory nerve conduction velocity (SNCV) compared to diabetic mice with wild type TNF- $\alpha$ expression [12]. To further characterize TNF- $\alpha$ role in diabetic neuropathy, neutralization studies were conducted using the TNF- $\alpha$ neutralizing anti-body, infliximab. Infliximab treatment following STZ injections recovered MNCV and SNCV losses, tail flick nociceptive behavior, and prevented a loss of epidermal nerve fibers compared to STZ-injected control animals [12]. Treatment with the TNF- $\alpha$ neutralizing antibody was also effective in reducing circulating TNF- $\alpha$ serum levels and TNF- $\alpha$ mRNA expression in the DRG back to control animal levels [12]. Thus, these knockout and neutralizing studies highlight a likely important role of TNF- $\alpha$ in the development of DN.

While, TNF- $\alpha$ stands out as a cytokine with the most evidence to support a role for cytokines in DN, research also suggests that chemokines and interleukins could also be involved. Moving forward, studies expanding on the correlative studies and moving towards a more mechanistic approach are needed.

\section{Cyclooxygenase}

Cyclooxygenase (COX) is the rate-limiting enzyme for prostaglandin synthesis and its inhibition is the target of most nonsteroidal anti-inflammatory drugs (NSAIDs). There are two COX isoforms identified as COX-1 and COX-2. COX-1 is constitutively expressed, while COX-2 has limited expression under normal conditions, but is induced in inflammatory conditions. The inducible nature of COX-2 makes it a good candidate for monitoring the inflammatory state of tissue. Diabetic rodent studies have revealed altered cyclooxygenase expression, including increased COX-2 protein levels in the sciatic nerve and sensory neurons of STZ injected rodents $[30,31]$. Insight into the role of COX-2 in the development of DN was explored using COX-2 deficient mice. These studies suggested that the absence of COX-2 protected STZ-injected animals against decreases in SNCV, MNCV, endoneurial blood flow, and intraepidermal nerve fiber density compared to nondiabetic mice $[31,32]$. The prevention of 
these characteristic DN symptoms supports a critical role COX-2 in DN. Importantly, the absence of COX-2 expression in COX-2 deficient diabetic mice was effective at mediating these effects on nerve function for up to 6 months following STZ injections [31]. These studies suggest an ongoing role for COX enzymatic activity in the development of DN.

\section{Therapeutic Treatments Targeting Inflammatory Mediators in Diabetic Neuropathy}

With the discovery that inflammatory mediators such as TNF- $\alpha$ are increased in DN, researchers began to focus on therapeutic treatments that could target these inflammatory mediators. In vitro experiments using monocytes from type 2 diabetic patients demonstrated increased expression of TNF- $\alpha$, IL-1, IL- 6 , and IL-8 compared to healthy controls and type 1 diabetic patients. Treatment of human monocytes from type 2 patients with the active form of vitamin $\mathrm{D}, 1,25$-dihydroxyvitamin D3, downregulates the mRNA expression of TNF- $\alpha$, IL-1, IL-6, and IL-8 [33]. While these in vitro experiments demonstrated the ability of therapeutics to target inflammatory mediators in DN, further in vivo experiments were needed. In vivo rodent experiments using the natural flavonoid, curcumin, dose-dependently decreased serum TNF- $\alpha$ levels and attenuated thermal hyperalgesia in STZ-treated mice $[28,34]$. The beneficial effect of curcumin treatment was enhanced with co-treatment with insulin [28]. Additional therapeutics capable of preventing inflammatory mediated events in rodent models included gliclazide, a sulfonylurea used in non-insulin dependent diabetes, and troglitazone, a thiazolidinedione. Both gliclazide and troglitazone attenuated TNF- $\alpha$ levels and improved MNCV in STZ-injected rats. These treatments also prevented decreases in myelinated fiber area, fiber density, and axon/myelin ratio in the tibial nerve of diabetic rats $[25,26]$. An additional therapeutic that was tested was the anti-oxidant, $\mathrm{N}$-acetylcysteine, which dose dependently improved TNF- $\alpha$ levels and MNCV in STZ-induced diabetic rats [27]. While all the mentioned therapeutics do not belong to a unifying drug class, they were all shown to have an effect on TNF- $\alpha$ levels and were able to help in preventing the development of DN.

NSAIDS are a classical therapeutic for inflammatory conditions and are available in both over-the-counter and prescription forms. As stated previously, this therapeutics' mechanism of action is targeted at inhibiting the enzyme COX. NSAIDS include both non-selective COX inhibitors such as ibuprofen and selective COX-2 inhibitors such as celecoxib. Animal studies exploring the therapeutic potential of these compounds in DN have begun to be explored following the discovery of increased COX-2 levels in peripheral tissues in DN models. Nonselective COX inhibitors have shown mixed results. Piroxicam was shown to statistically increase STZ-induced decreases in sensory neuron action potential amplitude [35]. The non-selective inhibitors, sulindac and indomethacin, corrected losses in sural and caudal sensory nerve conduction velocity of diabetic rodents compared to control mice $[36,37]$. While some non-selective COX inhibitors have shown to be an effective treatment option, flurbiprofen treatment alone induced decreases in MNCV. Thus, flurbiprofen treatment mimicked STZinduced changes and lacked the ability to alter STZ-induced changes on MNCV [30]. The authors suggested inhibiting the ubiquitously expressed COX-1 is responsible for these effects, indicating COX-1 may be responsible for maintaining neural function in rodents [30]. Given this observation, the use of selective COX-2 inhibitors was utilized to determine their effectiveness. Celecoxib treatment prevented MNCV and SNCV slowing [31], and meloxicam treatment was shown to protect against MNCV slowing and endoneurial blood flow deficits in diabetic rodents [30]. Further characterization through intrathecal administration of COX-2 inhibitors revealed a dose dependent attenuation of mechanical behavior [38]. Another interesting effect of selective inhibition of COX-2 through pharmacological or gene inactivation is a prevention of increased TNF- $\alpha$ expression in the sciatic nerve of STZ-induced diabetic rodents [31].

Limited corresponding research has been done in clinical studies. However, one patient study has been conducted evaluating NSAID treatment in diabetic patients, which demonstrated an improvement of neuropathy score with ibruprofen and sulindac treatment compared to placebo treatment of the same patients [39]. The interpretation of these results is limited because of the lack of a healthy age-matched control group. Comparisons in this study were only made between responders and non-responders among the DN patients. While NSAID treatment is a promising avenue to explore with a number of currently available treatment options, known side effects of this class of drugs would be prohibitive to long-term treatment. Although selective COX-2 inhibitors would prevent against gastrointestinal side effects, the cardiovascular effects of COX-2 inhibitors could be problematic, especially in a patient population already at increased risk for cardiovascular complications.

Therapeutic treatments aimed at targeting inflammatory mediators in DN thus far have shown effects when the therapeutic is administered at the time of DN induction. There is a lack of evidence to demonstrate their effectiveness after the development of $\mathrm{DN}$ in reversing any of the symptoms of $\mathrm{DN}$ such as reductions in nerve conduction velocities or nociceptive behavior. It is highly unlikely the initiation of therapeutic treatment would coincide with the initial development of diabetes in patients. Therefore, studies investigating the time course of antiinflammatory therapeutics are needed. While current studies haven't addressed reversal of $\mathrm{DN}$, a notable finding from a limited number of studies is that treatment effects were ineffective in initial time points, but instead the statistically significant beneficial effects were only evident after 12 weeks of treatment $[25,26]$. This temporal aspect may suggest the inflammatory component of $\mathrm{DN}$ might not develop until later time points. Therefore, administration of therapeutics at later time points might still be effective, but further studies are needed to validate these findings.

\section{Cytokine Expression Profile in Painful versus Painless Neuropathy}

One interesting component of $\mathrm{DN}$ is the presence of both painful and painless presentation in human and rodent studies. The cause of this presentation of both painful and painless neuropathies is unclear. One theory put forth by Uceyler and colleagues points toward differences in the cytokine expression profile. Their study evaluated both pro- and anti-inflammatory cytokine levels in painful and painless patient populations. The patient population for the study encompassed a number of neuropathic pain conditions, including diabetic neuropathy and chronic inflammatory demyelinating neuropathy. The authors were able to demonstrate that patients with painful neuropathy had 2-fold greater levels of TNF- $\alpha$ and IL-2 compared to controls and painless neuropathy patients. Another notable difference in the cytokine profile of this patient population was found when analyzing the painless patients samples which had statistically higher circulating levels of the anti-inflammatory cytokine, IL-10, twofold higher compared to controls [24]. These findings are interesting given the recent publication from Saleh and colleagues that showed a decrease in TNF- $\alpha$ levels in the DRG of STZ-injected rats [40]. These findings are in stark contrast to other papers that have demonstrated 
an increase in TNF- $\alpha$ levels in patients and diabetic rodent models [2228]. One reason for this discrepancy could be attributed to differences in the phenotypic expression of the nociceptive behavior in different models of DN. For example, previous studies have found diabetic rodents with decreased DRG TNF- $\alpha$ levels demonstrate hypoalgesic behavior [40], while diabetic rodents with increased DRG TNF-a levels display hyperalgesic behavior [12]. Whether this is consistent with all rodent DN models is unclear. However, support for increased TNF- $\alpha$ levels contributing to nociceptive behavior has been demonstrated in previous publications independent of a DN state [20,21]. These results would suggest that individuals with painful neuropathies have a predominantly pro-inflammatory cytokine expression profile, while patients with painless neuropathies are more susceptible to antinflammatory cytokine expression. In all likelihood, the predisposing factor that makes a patient susceptible to develop a painful versus painless neuropathic pain state is likely from differences in genetic make-up, but the mechanism responsible for these differences is unknown.

There are other possible confounding factors that could be contributing to the differences in cytokine levels in painful and painless neuropathy patients. For example, depression, which has also been shown to correspond with increased cytokine levels, could be contributing the differences witnessed. Uceyler and colleagues acknowledge in their conclusion that the painful neuropathy patients had higher depression scores than painless patients [24]. This assumption is made stronger with the evidence that higher TNF- $\alpha$ levels were present in depressed painful neuropathy patients compared to those without depression [41]. Whether depression is the determining factor for differences in cytokine levels, or if the decreases in quality of life resulting from increased pain itself are contributing to the depression is unclear. Another variable associated with the time of sample collection and duration of illness needs to be addressed. This variable was suggested by Empl and colleagues as contributing to the results of their study that found increased Schwann cell TNF- $\alpha$ expression in painful neuropathy patients compared to non-painful patients [42]. The authors noted that the painful neuropathy patients in their study had a shorter duration of illness, explaining this by the painful symptoms prompting patients to seek medical attention earlier. The validity of this concern stems from inflammatory mediators, such as TNF- $\alpha$, are often reported in the early stages of nerve injury, during time periods when the immune system is responding to the insult. Taking this into account, the initial immune response would be early and probably provide a minor contribution to when the samples were collected. In fact, there is no significant difference in the duration of illness in the painful and painless neuropathy patients from the previously cited study (76.14 \pm 24.17 months vs. $94.99 \pm 21.58$ months), and the duration of the illness would be well past an initial immune response [24]. Taking into account the outlined confounding variables that could be contributing, follow-up studies with a greater number of patients, particularly DN patients, and with samples from a number of time points would further validate these findings.

\section{Conclusions}

The increase of inflammatory mediators in a number of neuropathic pain states has been well documented. The potential role of these mediators in DN is just beginning to be explored, with TNF-a and COX-2 currently demonstrating the strongest involvement (for summary of current results refer to Table 1). Further investigation into additional inflammatory mediators such as interleukins and chemokines is warranted, including rodent studies to explore the

\begin{tabular}{|c|c|c|c|c|}
\hline Inflammatory Mediator & Level & Tissue & Model & Reference \\
\hline TLR4 & increase & monocyte & Type 1 patients & Devaraj et al. 2008 \\
\hline TLR2 & increase & monocyte & Type 1 patients & Devaraj et al. 2008 \\
\hline \multirow{3}{*}{ MCP1 } & increase & peripheral blood & Type 2 patients & Nomura et al. 2000 \\
\hline & increase & serum & Type 1 patients (multiple complications) & Guan et al. 2011 \\
\hline & increase & plasma and adipose & ob/ob diabetic mice & Sartipy and Loskutoff 2003 \\
\hline RANTES & increase & peripheral blood & Type 2 diabetic patients & Nomura et al. 2000 \\
\hline \multirow{10}{*}{ TNF- $\alpha$} & increase & plasma and macrophage & DN (painful compared to painless) & Purwata et al. 2011 \\
\hline & decrease & Lumbar DRG & STZ-induced rat model & Saleh et al. 2011 \\
\hline & increase & serum & STZ-induced mouse model & Yamakawa et al. 2011 \\
\hline & increase & DRG (mRNA) & STZ-induced mouse model & Yamakawa et al. 2011 \\
\hline & increase & serum & DN patients compared to diabetic-no neuropathy & Doupis et al. 2009 \\
\hline & increase & sciatic nerve & STZ-induced mouse model & Kellogg et al. 2007 \\
\hline & increase & sciatic nerve & STZ-induced rat model & Kellogg et al. 2007 \\
\hline & increase & serum & STZ-induced mouse model & Sharma et al .2007 \\
\hline & increase & serum & STZ-induced rat model & Qiang et al. 1998a,b \\
\hline & increase & serum & STZ-induced mouse model & Sharma et al .2006 \\
\hline \multirow{3}{*}{ COX-2 } & increase & sciatic nerve & STZ-induced rat model & Kellogg et al. 2007 \\
\hline & increase & sciatic nerve and DRG & STZ-induced mouse model & Kellogg et al. 2007 \\
\hline & increase & sciatic nerve & STZ-induced rat model & Pop-Busui et al. 2002 \\
\hline \multirow[t]{3}{*}{ IL-6 } & increase & sciatic nerve (mRNA) & STZ-induced mouse model & Bierhaus et al. 2004 \\
\hline & increase & sural nerve & DN patients compared to control & Bierhaus et al. 2004 \\
\hline & increase & DRG (mRNA) & STZ-induced mouse model & Yamakawa et al. 2011 \\
\hline IL-1a & increase & monocyte & Type 1 and 2 patients compared to controls & Giulietti et al. 2007 \\
\hline IL-8 & increase & monocyte & Type-2 patients compared to control & Giulietti et al. 2007 \\
\hline \multirow{3}{*}{ NF-KB } & increase & DRG, sciatic, sural nerve & STZ-induced mouse model & Toth et al. 2008 \\
\hline & increase & sural nerve & DN patients compared to control & Bierhaus et al. 2004 \\
\hline & increase & sciatic nerve & STZ-induced mouse model & Bierhaus et al. 2004 \\
\hline
\end{tabular}

Table 1: 
mechanisms by which these proteins could result in a number of DN symptoms, including nerve conduction velocity and epidermal innervation deficits. Further translational studies are needed to confirm findings in animal models, especially expanding on the therapeutic potential of targeting inflammatory mediators. Additionally, follow-up studies determining whether differences in cytokine profile expression continue when comparing painful versus painless diabetic neuropathy patients is an interesting aspect to explore. If similar results are evident in DN patients, it could be beneficial in determining enhanced personalized treatments for diabetic neuropathy patients.

\section{References}

1. Toth C, Brussee V, Cheng C, Zochodne DW (2004) Diabetes mellitus and the sensory neuron. J Neuropathol Exp Neurol 63: 561-573.

2. Bierhaus A, Schiekofer S, Schwaninger M, Andrassy M, Humpert PM, et al. (2001) Diabetes-associated sustained activation of the transcription factor nuclear factor-kappaB. Diabetes 50: 2792-2808.

3. Bierhaus A, Haslbeck KM, Humpert PM, Liliensiek B, Dehmer T, et al. (2004) Loss of pain perception in diabetes is dependent on a receptor of the immunoglobulin superfamily. J Clin Invest 114: 1741-1751.

4. Schiekofer S, Andrassy M, Chen J, Rudofsky G, Schneider J, et al. (2003) Acute hyperglycemia causes intracellular formation of CML and activation of ras, p42/44 MAPK, and nuclear factor kappaB in PBMCs. Diabetes 52: 621633.

5. Devaraj S, Dasu MR, Rockwood J, Winter W, Griffen SC, et al. (2008) Increased toll-like receptor (TLR) 2 and TLR4 expression in monocytes from patients with type 1 diabetes: further evidence of a proinflammatory state. J Clin Endocrinol Metab 93: 578-583

6. Toth C, Rong LL, Yang C, Martinez J, Song F, et al. (2008) Receptor for advanced glycation end products (RAGEs) and experimental diabetic neuropathy. Diabetes 57: 1002-1017.

7. Dasu MR, Devaraj S, Zhao L, Hwang DH, Jialal I (2008) High glucose induces toll-like receptor expression in human monocytes: mechanism of activation. Diabetes 57: 3090-3098.

8. Devaraj S, Dasu MR, Park SH, Jialal I (2009) Increased levels of ligands of Tolllike receptors 2 and 4 in type 1 diabetes. Diabetologia 52: 1665-1668.

9. Arbour NC, Lorenz E, Schutte BC, Zabner J, Kline JN, et al. (2000) TLR4 mutations are associated with endotoxin hyporesponsiveness in humans. Nat Genet 25: 187-191.

10. Rudofsky G, Reismann P, Witte S, Humpert PM, Isermann B, et al. (2004) Asp299Gly and Thr399lle genotypes of the TLR4 gene are associated with a reduced prevalence of diabetic neuropathy in patients with type 2 diabetes. Diabetes Care 27: 179-183.

11. Uceyler N, Kafke W, Riediger N, He L, Necula G, et al. (2010) Elevated proinflammatory cytokine expression in affected skin in small fiber neuropathy. Neurology 74: 1806-1813.

12. Yamakawa I, Kojima H, Terashima T, Katagi M, Oi J, et al. (2011) Inactivation of TNF \{alpha\} Ameliorates Diabetic Neuropathy in Mice. Am J Physiol Endocrinol Metab 301: 844-852.

13. Oh SB, Tran PB, Gillard SE, Hurley RW, Hammond DL, et al. (2001) Chemokines and Glycoprotein120 Produce Pain Hypersensitivity by Directly Exciting Primary Nociceptive Neurons. J Neurosci 21: 5027-5035.

14. White FA, Wilson NM (2008) Chemokines as pain mediators and modulators. Curr Opin Anaesthesiol 21: 580-585.

15. Sartipy P, Loskutoff DJ (2003) Monocyte chemoattractant protein 1 in obesity and insulin resistance. Proc Natl Acad Sci U S A 100: 7265-7270.

16. Nomura S, Shouzu A, Omoto S, Nishikawa M, Fukuhara S (2000) Significance of chemokines and activated platelets in patients with diabetes. Clin Exp Immunol 121: 437-443.

17. Guan R, Purohit S, Wang H, Bode B, Reed JC, et al. (2011) Chemokine (C-C motif) ligand 2 (CCL2) in sera of patients with type 1 diabetes and diabetic complications. PLoS One 6: e17822.

18. Doupis J, Lyons TE, Wu S, Gnardellis C, Dinh T, et al. (2009) Microvascular reactivity and inflammatory cytokines in painful and painless peripheral diabetic neuropathy. J Clin Endocrinol Metab 94: 2157-2163.

19. Sorkin LS, Xiao WH, Wagner R, Myers RR (1997) Tumour necrosis factor-alpha induces ectopic activity in nociceptive primary afferent fibres. Neuroscience 81 : 255-262.

20. Wagner R, Myers RR (1996) Endoneurial injection of TNF-alpha produces neuropathic pain behaviors. Neuroreport 7: 2897-2901.

21. Okamoto K, Martin DP, Schmelzer JD, Mitsui Y, Low PA (2001) Pro- and antiinflammatory cytokine gene expression in rat sciatic nerve chronic constriction injury model of neuropathic pain. Exp Neurol 169: 386-391.

22. Purwata TE (2011) High TNF-alpha plasma levels and macrophages iNOS and TNF-alpha expression as risk factors for painful diabetic neuropathy. J Pain Res 4: 169-175.

23. Navarro JF, Mora C, Muros M, Garcia J (2006) Urinary tumour necrosis factoralpha excretion independently correlates with clinical markers of glomerular and tubulointerstitial injury in type 2 diabetic patients. Nephrol Dial Transplant 21: $3428-3434$

24. Uceyler N, Rogausch JP, Toyka KV, Sommer C (2007) Differential expression of cytokines in painful and painless neuropathies. Neurology 69: 42-49.

25. Qiang X, Satoh J, Sagara M, Fukuzawa M, Masuda T, et al. (1998) Gliclazide inhibits diabetic neuropathy irrespective of blood glucose levels in streptozotocin-induced diabetic rats. Metabolism 47: 977-981.

26. Qiang X, Satoh J, Sagara M, Fukuzawa M, Masuda T, et al. (1998) Inhibitory effect of troglitazone on diabetic neuropathy in streptozotocin-induced diabetic rats. Diabetologia 41: 1321-1326.

27. Sagara M, Satoh J, Wada R, Yagihashi S, Takahashi K, et al. (1996) Inhibition of development of peripheral neuropathy in streptozotocin-induced diabetic rats with N-acetylcysteine. Diabetologia 39: 263-269.

28. Sharma S, Chopra K, Kulkarni SK (2007) Effect of insulin and its combination with resveratrol or curcumin in attenuation of diabetic neuropathic pain: participation of nitric oxide and TNF-alpha. Phytother Res 21: 278-283.

29. Satoh J, Yagihashi S, Toyota T (2003) The possible role of tumor necrosis factor-alpha in diabetic polyneuropathy. Exp Diabesity Res 4: 65-71.

30. Pop-Busui R, Marinescu V, Van Huysen C, Li F, Sullivan K, et al. (2002) Dissection of metabolic, vascular, and nerve conduction interrelationships in experimental diabetic neuropathy by cyclooxygenase inhibition and acetyl-Lcarnitine administration. Diabetes 51: 2619-2628.

31. Kellogg AP, Wiggin TD, Larkin DD, Hayes JM, Stevens MJ, et al. (2007) Protective effects of cyclooxygenase-2 gene inactivation against peripheral nerve dysfunction and intraepidermal nerve fiber loss in experimental diabetes. Diabetes 56: 2997-3005.

32. Kellogg AP, Pop-Busui R (2005) Peripheral nerve dysfunction in experimental diabetes is mediated by cyclooxygenase- 2 and oxidative stress. Antioxid Redox Signal 7: 1521-1529.

33. Giulietti A, van Etten E, Overbergh L, Stoffels K, Bouillon R, et al. (2007) Monocytes from type 2 diabetic patients have a pro-inflammatory profile. 1,25-Dihydroxyvitamin $\mathrm{D}(3)$ works as anti-inflammatory. Diabetes Res Clin Pract 77: 47-57.

34. Hingtgen SD, Tian X, Yang J, Dunlay SM, Peek AS, et al. (2006) Nox2containing NADPH oxidase and Akt activation play a key role in angiotensin II-induced cardiomyocyte hypertrophy. Physiol Genomics 26: 180-191.

35. Parry GJ, Kozu H (1990) Piroxicam may reduce the rate of progression of experimental diabetic neuropathy. Neurology 40: 1446-1449.

36. Zochodne DW, Ho LT (1992) The influence of indomethacin and guanethidine on experimental streptozotocin diabetic neuropathy. Can J Neurol Sci 19: 433 441.

37. Zochodne DW, Ho LT (1994) The influence of sulindac on experimental streptozotocin-induced diabetic neuropathy. Can J Neurol Sci 21: 194-202.

38. Matsunaga A, Kawamoto M, Shiraishi S, Yasuda T, Kajiyama S, et al. (2007) Intrathecally administered COX-2 but not COX-1 or COX-3 inhibitors attenuate streptozotocin-induced mechanical hyperalgesia in rats. Eur J Pharmacol 554: $12-17$.

39. Cohen KL, Harris S (1987) Efficacy and safety of nonsteroidal anti-inflammatory drugs in the therapy of diabetic neuropathy. Arch Intern Med 147: 1442-1444. 
40. Saleh A, Smith DR, Balakrishnan S, Dunn L, Martens C, et al. (2011) Tumor necrosis factor-alpha elevates neurite outgrowth through an NF-kappaBdependent pathway in cultured adult sensory neurons: Diminished expression in diabetes may contribute to sensory neuropathy. Brain Res 1423: 87-95.
41. Anisman $\mathrm{H}$, Merali Z, Poulter MO, Hayley S (2005) Cytokines as a precipitant of depressive illness: animal and human studies. Curr Pharm Des 11: 963-972.

42. Empl M, Renaud S, Erne B, Fuhr P, Straube A, et al. (2001) TNF-alpha expression in painful and nonpainful neuropathies. Neurology 56: 1371-1377. 